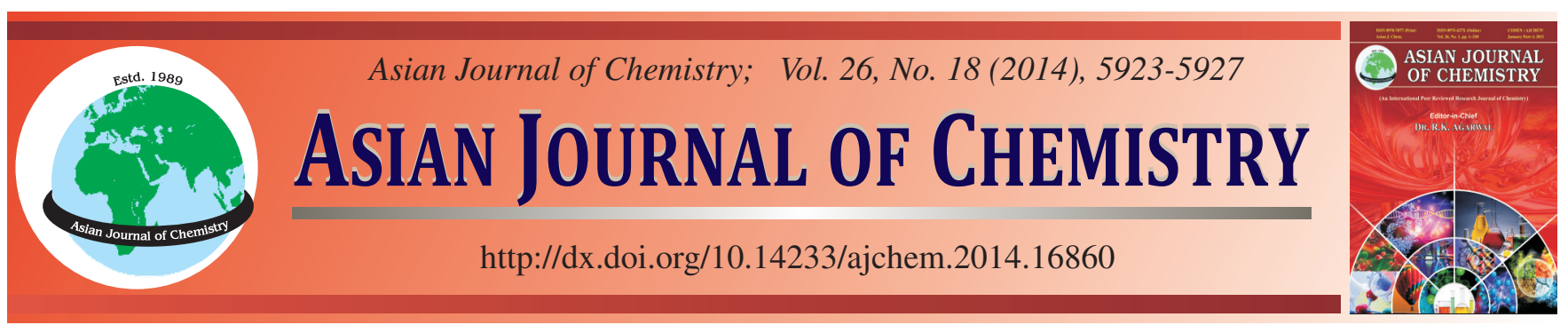

\title{
REVIEW
}

\section{Hydrophobically Modified Polyacrylamide Synthesis and Application in Water Treatment}

\author{
Yi Liao ${ }^{1,2}$, Huaili Zheng ${ }^{1,3, *}$, Li Dai ${ }^{1}$, Fengting Li $^{4}$, Guocheng Zhu ${ }^{1}$, Qingling Guan ${ }^{1}$, Yonguun Sun $^{1}$ and Xiaomin Tang ${ }^{1}$
}

${ }^{1}$ Key Laboratory of the Three Gorges Reservoir Region's Eco-Environment, State Ministry of Education, Chongqing University, Chongqing 400045, P.R. China

${ }^{2}$ Department of Architectural Engineering, Sichuan University of science and engineering, Zigong 643000, P.R. China

${ }^{3}$ National Centre for International Research of Low-Carbon and Green Buildings, Chongqing University, Chongqing 400045, P.R. China

${ }^{4}$ College of Environmental Science and Engineering, Tongji University, Shanghai 200092, P.R. China

*Corresponding author: Tel./Fax: +86 23 65120827, E-mail: zhl@cqu.edu.cn; ly-001-1980@163.com

Received: 11 December 2013

Accepted: 3 April 2014;

Published online: 1 September 2014;

AJC-15823

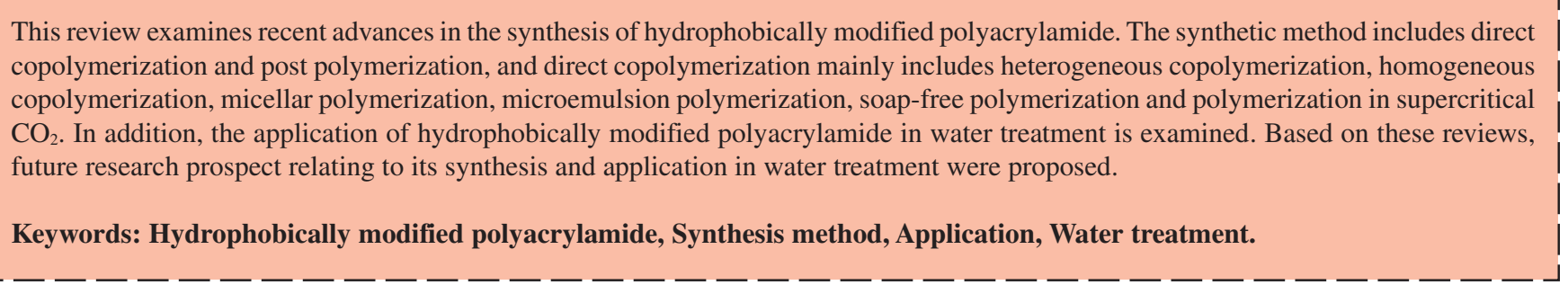

\section{INTRODUCTION}

In recent years, extensive studies in academic and industrial laboratories have focused on the development of watersoluble hydrophobically associated polymers ${ }^{1-6}$. The incorporation of a small amount of hydrophobic groups in a hydrophilic macromolecular chain can result in unique rheological characteristics such as enhanced thickening efficiency, shearthickening rheology, as well as improved temperature resistance, salt tolerance and shear tolerance in aqueous solution ${ }^{3,7-9}$. This ability of the system is conducive to its applications including cosmetics, drilling fluids, oil recovery, paints, as well as biotechnological and pharmaceutical applications and water treatment ${ }^{8,10,11}$. Generally, water-soluble hydrophobically associating polymers are prepared using acrylamide (AM) as hydrophilic monomer. Several experiments have shown that acrylamide is the monomer most suited for the manufacture of high molecular weight water-soluble polymers at polymer concentration below 1 mass $\%{ }^{12}$.

The best solvent for acrylamide is water. However, hydrophobic monomers do not normally dissolve in water. So the major challenge in the synthesis of hydrophobically modified polyacrylamide (HMPAM) originates from the mixing of both oil-soluble and water-soluble monomers in water.
This review examines recent advances in the synthesis of hydrophobically modified polyacrylamide. The synthetic method includes both direct synthesis via copolymerization and post polymerization. The basic principle and features of various methods were described and summarized. Meanwhile, the applications especially in water treatment are reviewed. Finally, the main conclusions and prospects on this issue have been simply examined.

Selection of monomers: In the process of preparing hydrophobically modified polyacrylamide, the hydrophilic monomer is acrylamide. The polymerization reaction of acrylamide in solution commonly could be expressed as Fig. 1. Meanwhile, hydrolyzed acrylamide or other hydrophilic comonomers such as acrylic acid and sodium acrylate have been used to increase viscosity of the system ${ }^{11,13,14}$. In addition, sulfonate-containing monomers including vinyl sulfonate, 4-vinyl benzene sulfonate and 2-acrylamido-2-methylpropanesulfonic acid (AMPS) have been used to replace acrylic acid and improve the salt sensitivity of the resulting copolymer $^{15-17}$. Moreover, N-vinyl pyrrolidinone (NVP) has sometimes been used to make the resulting copolymer more resistant to base-catalyzed hydrolysis of acrylamide ${ }^{18,19}$. Hydrophobic monomers are usually $\mathrm{N}$-alkyl (aryl)acrylamide ${ }^{7,20,21}$, acrylate or methacrylate ${ }^{22}$ and styrene ${ }^{23,24}$. Other hydrophobic monomers are mainly their derivatives ${ }^{25}$. Among these 
hydrophobic monomers, $\mathrm{N}$-alkyl acrylamide has become the most important hydrophobic monomer in laboratory and industrial filed, because they not only can be well copolymerized with acrylamide but also has a strong capability of resistant to hydrolysis. In addition, hydrophobic monomers containing fluorocarbons have also been prepared ${ }^{8,15,26,27}$. It was found that hydrophobic association occurring between fluorocarbon chains was stronger than the interactions of the corresponding hydrocarbon comonomers ${ }^{28,29}$. So far, the study about the fluorocarbon hydrophobically modified polyacrylamide derivant is still in initial stage and it need to be further research in many ways.

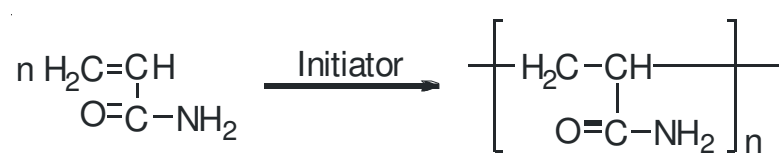

Fig. 1. Polymerization reaction of acrylamide in solution

Synthesis method: Generally, there are two kinds of methods to incorporate hydrophobic groups into water soluble polymer chains. The first kind of method is the direct copolymerization of water-soluble and hydrophobic monomers. The second kind of method is the modification of polymers after polymerization to introduce hydrophobic or hydrophilic groups ${ }^{15}$.

Copolymerization: Copolymerization is characterized by the chemical reaction between water-soluble and oil-soluble monomers with unsaturated bond under certain conditions. To achieve the copolymerization of acrylamide and hydrophobic monomers, the problems associated with the mixture of two kinds of monomers must be settled first. Fig. 2 shows the typical process of copolymerization of hydrophobically modified polyacrylamide. According to the various mixture methods of hydrophobic and hydrophilic monomers, the method of copolymerization can be divided into the following categories:

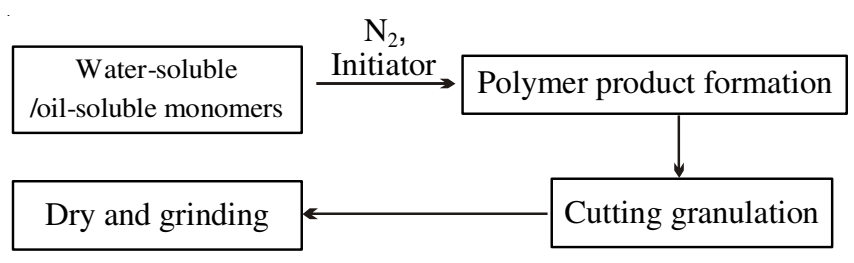

Fig. 2. Typical process of copolymerization of hydrophobically modified polyacrylamide

Heterogeneous copolymerization: The earliest method of preparing hydrophobically modified polyacrylamide is heterogeneous copolymerization, in which hydrophobic monomers is dispersed to finely dispersed state under mechanical stirring to copolymerize with hydrophilic monomers in water. Hill et al..$^{30}$ prepared a kind of hydrophobically modified polyacrylamide by heterogeneous copolymerization. In this process, ethylphenylacrylamide was dispersed as a fine powder in the aqueous solution of acrylamide under the mechanical stirring. As the conversion rate of reaction system increased, the solid particles were gradually consumed. The study showed that the final solution appeared quite homogeneous for a copolymer with a very low hydrophobe content (1 mol \%), while the solution appeared somewhat cloudy for a copolymer with a higher hydrophobe content (2-3 mol \%). In the case of heterogeneous copolymerization, a random hydrophobe distribution of copolymer was observed. There was no obvious improvement in the thickening properties observed in the resulting copolymer. A more hydrophobic monomer are required to increase the thickening properties of the resulting copolymer, and which would led to an insoluble copolymer. So the heterogeneous copolymerization does not seem to be a convenient way for preparing efficient hydrophobically modified polyacrylamide, and this method is now rarely used.

Homogeneous copolymerization: Homogeneous polymerization is carried out in an organic solvent or a water-based solvent mixture in which both hydrophobic and hydrophilic monomers are soluble. Although the dispersion on a molecular scale can be achieved in this process ${ }^{31}$, there are other problems associated with this technique. For example, though a common solvent can be found for the monomers, it is unlikely that the polymer will also be soluble in such a medium ${ }^{32-34}$. Only a few examples are reported in which the copolymer is maintained in solution homogeneous polymerization ${ }^{30,35}$. Generally, the typical common solvents for the two monomers are methanol, ethanol, chloroform, acetone and formamide, etc. When these organic (co) solvent are used, there is another constraint due to the possibility of chain transfer reactions leading to a lowering of the molecular weights ${ }^{30}$. In addition, a lot of research has shown that a random microstructure of the resulting polymer is obtained by the homogeneous copolymerization process ${ }^{30,32-34}$. Mean while there is no obvious improvement in the hydrophobically association action of the resulting polymer prepared by homogenous copolymerization in aqueous solution ${ }^{30}$.

Micellar polymerization: One of the most effective methods to prepare hydrophobically modified polyacrylamide is micellar polymerization technique. The first reports on micellar copolymerization of hydrophobically modified polyacrylamide appeared simultaneously ${ }^{25,31}$. In the micellar copolymerization process, the hydrophobic monomer is solubilized within surfactant micelles, whereas the hydrophilic monomer is dissolved together with the initiator in the aqueous continuous medium. The copolymerization reaction occurs in both the continuous phase and the micelle phase induced by the free radicals (Fig. 3). Although the reaction mixture is optically transparent, it is actually a microheterogeneous system. The micelles in this system behave as microdomains with a high local hydrophobe concentration. This process yields a copolymer with a blocky structure ${ }^{36,37}$.

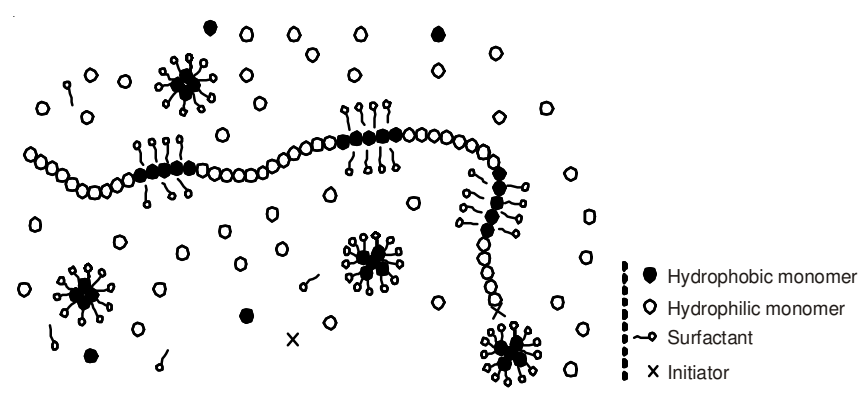

Fig. 3. Schematic presentation of micellar copolymerization ${ }^{37,38}$ 
The common initiators in micellar polymerization is potassium persulfate ${ }^{9,14,20,31}$, ammonium persulfate ${ }^{39-41}, 2,2$-azobis2-methylpropionitrile $(\mathrm{AIBN})^{15,42}$, etc. Typical surfactants for neutral or anionically charged hydrophobic polymers are sodium dodecyl sulfate (SDS) or nonionic ethoxylates. For cationic polymers, either cationic or nonionic surfactants are used. The fluorinated surfactants are needed when the hydrophobic monomer are fluorinated hydrophobe ${ }^{28,39,40}$. Among various surfactants, sodium dodecyl sulfate is the most frequently used surfactant $t^{14,30,41,43}$ since it is readily available in a pure form and the resulting polymer with higher molecular weight can be prepared. Generally, typical surfactant concentrations range from 1 to 6 mass $\%$. In earlier research, the surfactant content was thought to be adjusted only by taking into account the efficiency in solubilizing the hydrophobic monomer. But it is now well established that the surfactant content has another important role since it can strongly affect the microstructure of the resulting copolymer ${ }^{37,43-45}$.

Although the micellar polymerization is one of the most effective methods to prepare hydrophobically modified polyacrylamide, it also has some shortcomings as follows ${ }^{15,30,46}$ : Firstly, because of the chain transfer effects of surfactants and impurity within the surfactants, the resulting copolymer with low molecular weights would be obtained; secondly, a complex post treatment processes are required to remove the surfactants in order to purify the polymer product.

Microemulsion polymerization: Microemulsion is an optically transparent, thermodynamically stable and isotropic solution consisting of water, oil and a surfactant. When the reaction system is rich in water, the oil phase is uniformly dispersed in the form of small droplets in the continuous water phase and the oil-in-water $(\mathrm{O} / \mathrm{W})$ microemulsion is formed ${ }^{31,47}$. On the contrary, inverse microemulsion (water-in-oil type, $\mathrm{W} / \mathrm{O}$ ) is conducted with the W/O type emulsifier. In this system, oil phase is a continuous phase and water phase is uniformly dispersed within them. Then the system is initiated by the oilsoluble initiator or water-soluble initiator ${ }^{48,49}$.

In general, the copolymers with block structure can be obtained in $\mathrm{O} / \mathrm{W}$ microemulsion polymerization and more importantly, the hydrophobic length incorporated into the polymer chain can be regulated at pleasure. Besides, the copolymers prepared in W/O microemulision polymerization exhibit significant thickening effect at low oil-monomer concentration. And the copolymer prepared in this process shows better performances in salt-resistance and shear-resistance than the copolymer prepared in micellar polymerization.

However, there are some disadvantages for this technology. The main problem is the large quantities of surfactant needed to stabilize the system (usually $10 \%$ of the total mass). This will restrict the potential use of microemulsion polymerization because high solid contents and low surfactant amounts are usually desirable for most applications $\mathrm{s}^{50}$.

Soap-free polymerization: In order to improve the shortcomings caused by the surfactants in micellar polymerization, the soap-free polymerization was established. In this process, a micelle-forming polymerizable surfactant is used instead of the hydrophobic monomer in micellar polymerization ${ }^{46,51-54}$. Polymerizable surfactant is a kind of functional surfactant. It is called as surface-active monomer in scientific literatures, and often shortened to "surfomers" 15,54 . Their molecules contain both amphiphilic structure (including hydrophobic tail and hydrophilic head group) and polymerizable vinyl double bonds $s^{55}$. The special molecular architecture help them not only have surface activity like common surfactant but can also be used as a monomer or comonomer for polymerization. The reaction can be carried out by free radical polymerization in water without additional surfactant. In this process, the surfomer can be incorporated into the backbone in microblock manner by controlling the copolymerization conditions ${ }^{46,56}$ and hydrophobically modified polyacrylamide with strong intermolecular hydrophobic association can be obtained ${ }^{46}$.

The soap-free polymerization is the very effective way for preparing hydrophobically modified polyacrylamide with high molecular weight at present. Compared with other polymerization methods, the soap-free polymerization can eliminate pollutions to environment caused by the emulsifier, overcome the defects of the resulting copolymer caused by the residual emulsifier, simplify the reaction conditions and be conducive to the industrial production ${ }^{57}$.

Polymerizations in supercritical $\mathbf{C O}_{2}$ : Preparing hydrophobically modified polyacrylamide in supercritical $\mathrm{CO}_{2}$ is a very effective and novel method at present. DeSimone et al.$^{58}$ first reported the homogeneous polymerization of 1,1 dihydroperfluorooctyl acrylate in supercritical $\mathrm{CO}_{2}$ which substitutes the freon as commonly used solvent. From then on, supercritical $\mathrm{CO}_{2}$ as an alternative to conventional organic solvents has attracted increasing attention in recent years ${ }^{59-62}$. For preparing hydrophobically modified polyacrylamide, the supercritical $\mathrm{CO}_{2}$ is used as the solvent to dissolve both oilsoluble monnomer and water-soluble monomer ${ }^{63,64}$. And the mixture of two monomers on a molecular scale can be achieved in this process.

The main advantage of this method is as follows ${ }^{59,62}$ : Firstly, compared with the traditional solvents, the supercritical $\mathrm{CO}_{2}$ is a more environmentally friendly medium. It can eliminate the substantial harm by the use of volatile organic solvents on the manufacture and processing of commercial polymer products. In addition, because $\mathrm{CO}_{2}$ is an ambient gas, it can be easily recycled after use as a solvent to avoid any contribution to greenhouse effects. Secondly, $\mathrm{CO}_{2}$ is naturally occurring and abundant. It not only exists in nature throughout the world, but also is generated in large quantities as a byproduct in ammonia, hydrogen and ethanol plants and in electrical power generation stations that burn fossil fuels. Thirdly, $\mathrm{CO}_{2}$ has an easily accessible critical point with a $T c$ of $31.1^{\circ} \mathrm{C}$ and a $P c$ of 73.8 bar. Fourthly, the resulting products are virtually free from contamination and are easily obtained as dry powder just by releasing $\mathrm{CO}_{2}$ at the end of the reaction. In particular, because the oil-soluble monomers including amorphous fluoropolymers and silicones have good solubility in supercritical $\mathrm{CO}_{2}$, a large number of these hydrophobic groups can be incorporated into the molecular chain of polyacrylamide. Other methods are difficult to achieve the same effect.

However, since this method starts late with a short history, the study in many respects is still in a primary stage and there is less study on the industrialization and marketization of this technology. 
Post polymerization modification: Hydrophobically modified polyacrylamide can also be obtained by the postpolymerization reaction ${ }^{1,2}$. In this system, hydrophobically modified polyacrylamide have been prepared by incorporating hydrophobic (hydrophilic) groups into the hydrophilic (hydrophobic) polymer after the polymerization process. The reaction commonly occurred in homogenous medium. The main advantage of this method is that commercially available polymers can be used as starting material. However, the main drawback of this approach is that reactions are not easily carried out because of the problems associated with the mixing and reaction homogeneity caused by the viscous polymer solutions ${ }^{15}$.

Application in water treatment: The incorporation of a few hydrophobic groups into a hydrophilic macromolecular chain will lead to the system with unique rheological characteristics in aqueous solution. When above a certain polymer concentration, the hydrophobic moities will associate and build a transitory three-dimensional network that induces a strong increase in viscosity ${ }^{6,50}$. So there is a wide application prospect in fields including cosmetics, drilling fluids, oil recovery, paints, as well as biotechnological and pharmaceutical applications and water treatment, etc. Among them, hydrophobically modified polyacrylamide used in water treatment has been developed rapidly in recent years ${ }^{65-69}$.

Anionic or cationic polyacrylamide has been widely used as a kind of organic flocculant in water treatment and sludge dewatering process due to its effect of charge neutralization and absorption bridge $\mathrm{e}^{70}$. It has outstanding advantages such as low dosage demand, high efficiency, good solubility and pollution-free. With the incorporation of hydrophobic groups into the polymer molecular structure, the hydrophobic interaction between the molecular chains of the polymer is enhanced. Meanwhile the interaction between the polymer and organic matter get stronger and the hydrophilicity of flocs is reduced, thus a better flocculation effect can be obtained. Therefore, the charge neutralization effect, adsorption bridging effect and hydrophobic association effect can work together to contribute to the pollutants removal in water treatment and sludge dewatering ${ }^{65}$. Besides the removal efficiency of common pollutants such as suspended matter, sludge and sewage can be improved by an addition of hydrophobically modified polyacrylamide ${ }^{67,68}$, there are some other examples with respect to the special wastewater. For example, the characteristic of cationic group and hydrophobic group enable high oil removal efficiency on oily wastewater ${ }^{67,71}$, while some special hydrophobic groups such as aromatic compounds are conducive to the dyes removal in dye wastewater.

Conclusion and perspectives: This paper reviews the major advances achieved in the field of synthetic methods for hydrophobically modified polyacrylamide. One major difficulty in the synthesis of such polymer originates from the mixing and reacting oil-soluble and water-soluble monomers. The synthetic methods can be divided into two categories i.e., direct copolymerization and post polymerization method. Among the various synthetic methods, micellar polymerization is the most common method. The features of various synthesis methods are reviewed. It also briefly describes the application of hydrophobically modified polyacrylamide in water treatment.
At present, water treatment in China faced with many challenges, such as more complicated composition of wastewater as well as the strengthen of the people's consciousness of environment protection. Flocculation process is an important treatment technology with a wide range of applications in water treatment facilities. Flocculant as the core of flocculation process plays increasingly important role in water treatment. However, there are some shortcomings for current polyacrylamide such as the product of a single, low molecular weight and poor solubility. Therefore, research on flocculation should be concentrated in the synthesis of new, high performance and multifunctional type coagulant to improve the flocculation performance. Hydrophobically modified polyacrylamide is a new type flocculant and its flocculation mechanisms include not only charge neutralization and adsorption bridging in common flocculant, but also hydrophobic association. Although the research and application of such polymer in water treatment has been gotten fast development, profound researches in the synthesis process involving the optimization of reactive monomer, increases of molecular weight, cost reduction and improvement of the polymer performance are required in the future.

\section{Abbreviation}

HMPAM:Hydrophobically modified polyacrylamide

AM: Acrylamide

AMPS: 2-Acrylamido-2-methyl propanesulfonic acids

NVP: $\quad$ N-vinyl pyrrolidinone

AIBN: 2, 2-Azobis-2-methylpropionitrile

SDS: $\quad$ Sodium dodecyl sulfate

O/W: Oil-in-water

W/O: Water-in-oil

\section{ACKNOWLEDGEMENTS}

The authors are grateful for the financial support provided by the National Natural Science Foundation of China (Project No. 21177164) and the 111 Project (Project No. B13041).

\section{REFERENCES}

1. Y. Feng, L. Billon, B. Grassl, A. Khoukh and J. François, Polymer, 43, 2055 (2002).

2. Y. Feng, L. Billon, B. Grassl, G. Bastiat, O. Borisov and J. François, Polymer, 46, 9283 (2005).

3. D. Hourdet, G. Ducouret, S. Varghese, M.V. Badiger and P.P. Wadgaonkar, Polymer, 54, 2676 (2013).

4. H. Chen, X.Y. Wu, Z.B. Ye, L.J. Han and P.Y. Luo, Acta Phys. Chim. Sin., 28, 903 (2012).

5. Y.J. Guo, J.X. Liu, X.M. Zhang, R.S. Feng, H.B. Li, J. Zhang, X. Lv and P.Y. Luo, Energy Fuels, 26, 2116 (2012).

6. H. Chen, Z. Ye, L. Han and P. Luo, J. Appl. Polym. Sci., 123, 2397 (2012).

7. G. Yahaya, A. Ahdab, S. Ali, B. Abu-Sharkh and E. Hamad, Polymer, 42, 3363 (2001).

8. P. Li, Y. Shen and X. Yang, Polym. Bull., 67, 961 (2011).

9. P. Zhang, Y. Wang, W. Chen, H. Yu, Z. Qi and K. Li, J. Solution Chem., 40, 447 (2011).

10. Y. Umar, H.A. Al-Muallem, B. Abu-Sharkh and S.A. Ali, Polymer, 45, 3651 (2004).

11. W.L. Kang, X.Z. Wang, X.Y. Wu, L.W. Meng, S.R. Liu, B. Xu and X.H. Shan, Polym. Eng. Sci., 52, 2688 (2012).

12. D.A.Z. Wever, F. Picchioni and A.A. Broekhuis, Prog. Polym. Sci., 36, 1558 (2011). 
13. I. Blagodatskikh, O. Vasil'eva, E. Ivanova, S. Bykov, N. Churochkina, T. Pryakhina, V. Smirnov, O. Philippova and A. Khokhlov, Polymer, 45, 5897 (2004).

14. Y. Li and J.C. Kwak, Langmuir, 18, 10049 (2002).

15. K.C. Taylor and H.A. Nasr-El-Din, J. Petrol. Sci. Eng., 19, 265 (1998).

16. J. Bock and P.L. Valint, US Patent 4730028 (1988).

17. L. Jia, L. Yu, R. Li, X. Yan and Z. Zhang, J. Appl. Polym. Sci., 130, 1794 (2013).

18. D.N. Schulz, E. Berluche, J.J. Maurer and J. Bock, US Patent 4663408 (1987).

19. J. Bock, P.L. Valint and S.J. Pace, US Patent 4702319 (1987).

20. E. Volpert, J. Selb and F. Candau, Macromol., 29, 1452 (1996).

21. E.J. Regalado, J. Selb and F. Candau, Macromol., 32, 8580 (1999).

22. C.L. McCormick, J. Middleton and D. Cummins, Macromol., 25, 1201 (1992).

23. I. Lacík, J. Selb and F. Candau, Polymer, 36, 3197 (1995).

24. S. Biggs, A. Hill, J. Selb and F. Candau, J. Phys. Chem., 96, 1505 (1992).

25. S. Evani, US Patent 4432881 (1984).

26. J.-F. Berret, D. Calvet, A. Collet and M. Viguier, Curr. Opin. Colloid Interface Sci., 8, 296 (2003).

27. G.H. Li, X.M. Gong, C. Wang, N. Li and Z.K. Wang, J. Polym. Res., 20, (2013); doi:10.1007/s10965-013-0200-y.

28. Y.X. Zhang, A.H. Da, G. Butler and T. Hogen-Esch, J. Polym. Sci. Pol. Chem., 30, 1383 (1992).

29. R.C. Liu and F.M. Winnik, J. Photochem. Photobiol. A., 178, 208 (2006).

30. A. Hill, F. Candau and J. Selb, Macromol., 26, 4521 (1993).

31. S.R. Turner, D.B. Siano and J. Bock, US Patent 4520182 (1985).

32. J.J. Effing, I.J. McLennan, N.M. van Os and J.C. Kwak, J. Phys. Chem., 98, 12397 (1994).

33. S.A. Ezzell, C.E. Hoyle, D. Creed and C.L. McCormick, Macromol., 25, 1887 (1992).

34. S.A. Ezzell and C.L. McCormick, Macromol., 25, 1881 (1992).

35. H. Jiu, B. Gao and X. Cao, Acta Polym. Sin., 438 (2002).

36. K. Branham, D. Davis, J. Middleton and C.L. McCormick, Polymer (Guildf.), 35, 4429 (1994).

37. F. Candau and J. Selb, Adv. Colloid Interface Sci., 79, 149 (1999).

38. D. Wever, F. Picchioni and A. Broekhuis, Prog. Polym. Sci., 36, 1558 (2011).

39. F. Hwang and T. Hogen-Esch, Macromol., 28, 3328 (1995).

40. Y. Zhang, C. Wu, Q. Fang and Y.-X. Zhang, Macromol., 29, 2494 (1996).

41. Y.A. Shashkina, Y.D. Zaroslov, V. Smirnov, O. Philippova, A. Khokhlov, T. Pryakhina and N. Churochkina, Polymer, 44, 2289 (2003).

42. F.M. Winnik, H. Ringsdorf and J. Venzmer, Langmuir, 7, 905 (1991).

43. W. Xue, I.W. Hamley, V. Castelletto and P.D. Olmsted, Eur. Polym. J., 40, 47 (2004).

44. J. Ma, P. Cui, L. Zhao and R. Huang, Eur. Polym. J., 38, 1627 (2002).
45. V. Castelletto, I.W. Hamley, W. Xue, C. Sommer, J.S. Pedersen and P.D. Olmsted, Macromol., 37, 1492 (2004).

46. B. Gao, L. Jiang and K. Liu, Eur. Polym. J., 43, 4530 (2007).

47. S.R. Turner, D.B. Siano and J. Bock, US Patent 4521580 (1985)

48. F.W. Stanley Jr., US Patent 4524175 (1985)

49. H. Shang, J. Liu, Y. Zheng and L. Wang, J. Appl. Polym. Sci., 111, 1594 (2009).

50. F. Candau, M. Pabon and J.-Y. Anquetil, Colloids Surf. A, 153, 47 (1999).

51. D. Schulz, J. Kaladas, J. Maurer, J. Bock, S. Pace and W. Schulz, Polymer, 28, 2110 (1987).

52. M.C. Kramer, J.R. Steger, Y. Hu and C.L. McCormick, Macromol., 29, 1992 (1996).

53. B. Gao, H. Guo, J. Wang and Y. Zhang, Macromol., 41, 2890 (2008).

54. W.B. Li, H.Y. An, Y. Tan, C.G. Lu, C. Liu, P.C. Li, K. Xu and P.X. Wang, Soft Matter, 8, 5078 (2012).

55. M. Summers, J. Eastoe, S. Davis, Z. Du, R.M. Richardson, R.K. Heenan, D. Steytler and I. Grillo, Langmuir, 17, 5388 (2001)

56. G. Tuin, F. Candau and R. Zana, Colloids Surf. A, 131, 303 (1998).

57. E.E. Kathmann, L.A. White and C.L. McCormick, Macromolecules, 30, 5297 (1997).

58. J. DeSimone, Z. Guan and C. Elsbernd, Science, 257, 945 (1992).

59. J.L. Kendall, D.A. Canelas, J.L. Young and J.M. DeSimone, Chem. Rev., 99, 543 (1999).

60. H. Hu, T. He, J. Feng, M. Chen and R. Cheng, Polymer, 43, 6357 (2002).

61. H. Hu, M. Chen and R. Cheng, Polymer, 44, 341 (2003).

62. Z. Bin, H. Hu, M. Chen and W. Liu, Colloid Polym. Sci., 282, 1228 (2004).

63. T. He, L.-L. Yu, M.-C. Chen, H.-Q. Hu and S.-H. Lu, Polym. Mater. Sci. Eng., 21, 92 (2005).

64. L. Zheng, Y. Zheng, X. Xi and K. Chen, J. Northwest Univ., 38, 233 (2008).

65. Z.L. Yang, B.Y. Gao, C.X. Li, Q.Y. Yue and B. Liu, Chem. Eng. J., 161, 27 (2010).

66. H. Ren, Y. Li, S. Zhang, J. Wang and Z. Luan, Colloids Surf. A, 317, 388 (2008)

67. H.Z. Zhao, Z.K. Luan, B.Y. Gao and Q.Y. Yue, J. Appl. Polym. Sci., 84, 335 (2002)

68. H. Ren, W. Chen, Y. Zheng and Z. Luan, React. Funct. Polym., 67, 601 (2007).

69. K.E. Lee, N. Morad, B.T. Poh and T.T. Teng, Desalination, 270, 206 (2011).

70. T.P. Nguyen, N. Hilal, N.P. Hankins and J.T. Novak, Desalination, 227, 103 (2008).

71. S. Bratskaya, V. Avramenko, S. Schwarz and I. Philippova, Colloids Surf. A, 275, 168 (2006). 